\title{
Directing transport by polarized radiation in presence of chaos and dissipation
}

\author{
A.D. Chepelianskii ${ }^{(a)}$ and D.L. Shepelyansky $(b) *$ \\ ${ }^{(a)}$ Ecole Normale Supérieure, 45, rue d'Ulm, 75231 Paris Cedex 05, France \\ ${ }^{(b)}$ Laboratoire de Physique Théorique, UMR 5152 du CNRS, \\ Université P. Sabatier, 31062 Toulouse Cedex 4, France
}

(Dated: February 27, 2004)

\begin{abstract}
We study numerically the dynamics of particles on the Galton board of semi-disk scatters in presence of monochromatic radiation and dissipation. It is shown that under certain conditions the radiation leads to appearance of directed transport linked to an underlining strange attractor. The direction of transport can be efficiently changed by radiation polarization. The experimental realization of this effect in asymmetric antidot superlattices is discussed.
\end{abstract}

PACS numbers: 05.45.Ac, 05.60.-k, 72.20.Ht

A great challenge for the future technology on microscopic scale is the ability to control transport created by external energy sources in presence of dissipation and noise. These sources may drive a system out of equilibrium and generate a transport which direction is related to the system configuration in a rather nontrivial way. Such a directed transport appears in Brownian motors, or ratchets, which now attract a great interest of the community [1, 2, 3]. At present the technological progress allowed to observe ratchets experimentally in a variety of systems including semiconductor heterostructures [4], vortices in Josephson junction arrays [5, 6], cold atoms [], macroporous silicon membranes [8] and other systems. Biological applications of Brownian motors are also of primary importance 1, 2, 3].

The theoretical studies of ratchets have led to a number of interesting results (see [2] and Refs. therein) including such unexpected phenomena as absolute negative mobility when the transport is directed against an applied force [9]. However, up to now the studies of ratchets have been done mainly [10] in the limit of strong dissipation neglecting the effects of second time derivative [1, 2] or, in opposite, only the Hamiltonian dynamics has be analyzed 11, 12]. In this Letter we consider an intermediate case with a moderate or weak dissipation in a two-dimensional dynamical system driven by monochromatic polarized radiation. Without dissipation the dynamics is completely chaotic and is characterized by a homogeneous diffusion. Surprisingly, the dissipation related to friction leads to appearance of a directed transport which direction can be changed efficiently by polarization of radiation.

Our dynamical model describes the motion of noninteracting particles on the modified Galton board in presence of friction and polarized monochromatic radiation. The board of rigid disks on a triangular lattice had been introduced by Galton in 1889 to analyze the dynamics of particles elastically colliding with disks [13]. In absence of friction and radiation the dynamics is completely chaotic as it had been proven by Sinai (see e.g. 14]). To break the symmetry of the Galton board we replace disks by semi-disks oriented in the $x$ direction as shown in Fig. 1.

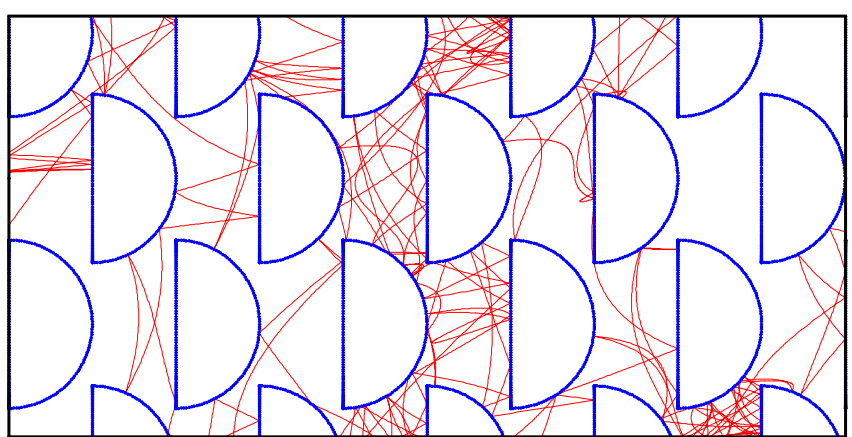

FIG. 1: (color online) A chaotic trajectory (red/grey curve) on the Galton board of semi-disks (blue/black) at radiation with $f=4, \theta=\pi / 8, \omega=1.5$ and dissipation $\gamma=0.1$. The trajectory starts near $x=y=0$, the region shown corresponds to $-27<x<-17,-10<y<-5$. Same trajectory on a large scale is shown in Fig. 3 .

Such a system has only translation and reflection $y \rightarrow-y$ symmetries.

We analyze the dynamics of particles on this modified Galton board under the influence of a linearly polarized radiation which gives a force directed by angle $\theta$ to $x$ axis: $\mathbf{f}=f(\cos \theta, \sin \theta) \cos \omega t$. In addition a particle experiences a friction force $\mathbf{F}_{f}=-\gamma \mathbf{v}$ directed against its velocity, and elastic collisions with semi-disks. A typical example of a chaotic trajectory in this regime is shown in Fig. 1. Without friction the dynamics is chaotic and a diffusive spreading in $x$ and $y$ directions takes place as well as a diffusive energy growth induced by $a c$-driving. However, due to the time reversibility, a directed transport is not possible in absence of friction. The introduction of friction breaks the time inversion symmetry and creates a directed transport.

To study numerically the dynamics of this model, we fix the disk radius $r_{d}=1$ and the particle mass $m=1$ so that the system is characterized only by the distance $R$ between disk centers. In the following we consider the case of compact semi-disk board with $R=2$ (a moderate variation of $R$ does not change qualitatively the dynamics properties). Following the approach of [15], the 


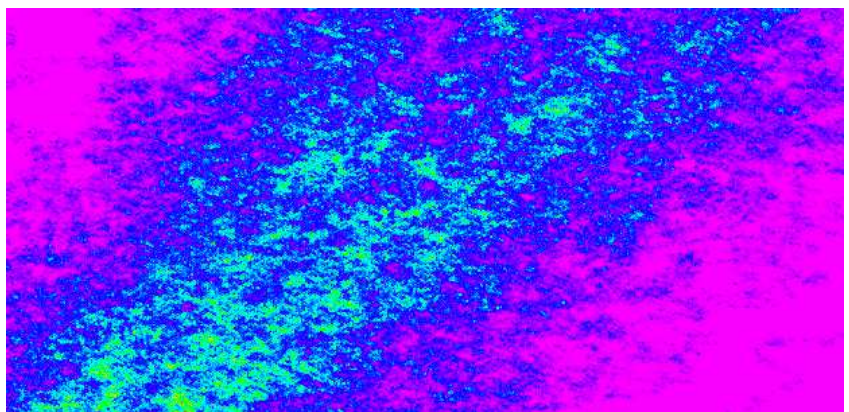

FIG. 2: (color online) Density distribution in the region $-400 \leq x \leq-100,0 \leq y \leq 600$ averaged over 200 trajectories in a time interval $0 \leq t \leq 2 \cdot 10^{4}$ for parameters of Fig. 1 . Initially all trajectories are distributed near $x=y=0$ with random velocity directions and $v^{2}=1$. For convenience the figure is clockwise rotated on $\pi / 2$, density is proportional to color changing from zero (rose/black) to maximum (green/white).

particle dynamics is simulated numerically by using the exact solution of Newton equations between collisions, and by determining the collision points with the rapidly converging Newton algorithm. This way at $f=0, \gamma=0$ the total energy is conserved with a relative precision of $10^{-14}$. A typical example of an average density distribution obtained from a simulation of many trajectories is shown in Fig. 2. In spite of chaos the dissipation leads to an average directed transport of particles moving under angle $\psi \approx 2.3$ to $x$-axis.

The direction of transport depends on the polarization of radiation as it is shown in Fig. 3 for typical trajectories computed on large time and space scales. The average velocity of directed flow can be written as $\mathbf{v}_{f}=v_{f}(\cos \psi, \sin \psi)$. For radiation polarized along $x$ $(\theta=0)$ the average transport goes along $-x$ direction $(\psi=\pi)$ while for radiation polarized along $y(\theta=\pi / 2)$ the direction of transport is inversed $(\psi=0)$. The fact that the particles move along $x$-axis for these $\theta$ values is in agreement with the symmetry properties of the semidisks board. However, in general the flow direction depends in a nontrivial way on the chaotic dynamics in presence of radiation and dissipation 16. By changing the polarization angle $\theta$ between 0 and $\pi / 2$ it is possible to change smoothly the angle of directed flow $\psi$ from $\pi$ to 0 (see Figs. 3,4). The values of $v_{f}, \psi$ and average velocity square $v^{2}=\left\langle v_{x}^{2}+v_{y}^{2}\right\rangle$ are determined from one long trajectory (up to times with $\omega t \sim 10^{7}$ ) or from averaging over 10 shorter orbits. The functional dependence $\psi(\theta)$ found in this way is shown in Fig. 4. It varies moderately with system parameters but, in average, can be approximated by a linear relation $\psi=\pi-2 \theta$. We note that in presence of radiation the directionality properties of transport are much more rich compared to the case of static applied force where for disks the chaotic transport simply follows the force direction [15, 18].

To understand the properties of the directed transport

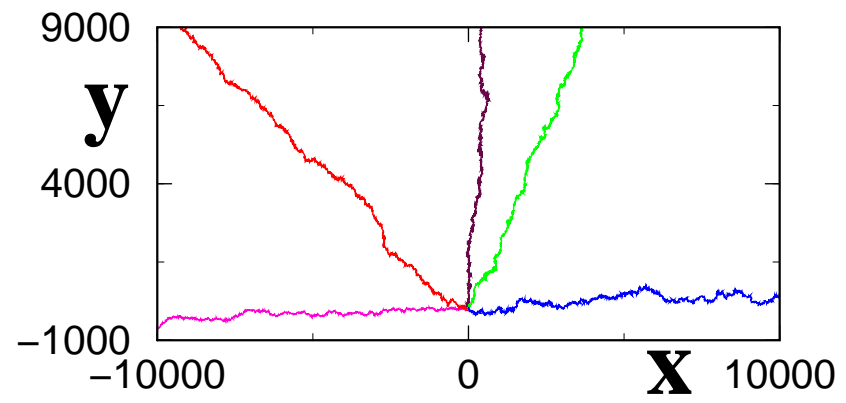

FIG. 3: (color online) Directed transport for one trajectory at various polarizations of radiation $\theta=0, \pi / 8$ (same trajectory as in Fig. 1), $0.21 \pi, \pi / 4, \pi / 2$ (from left to right clockwise) at $f=4, \omega=1.5, \gamma=0.1$.

let us start from the analysis of its averaged characteristics. It is clear that in presence of chaos ac-driving leads to an effective heating increasing the particle kinetic energy $E=v^{2} / 2$. This growth is stopped by energy dissipation induced by friction. The balance of these two processes gives an average energy $E$ of motion which due to chaos and dissipation takes place on a strange attractor [17]. This energy can be find on the basis of following estimates. Indeed, from the Newton equations of motion: $\dot{E} \sim v \dot{v} \sim f v \cos \omega t$. Hence, the diffusion rate in energy $D_{E}=(\Delta E)^{2} / t \sim \dot{E}^{2} \tau \sim f^{2} v l$ where the mean-free path $l \sim R \sim 1$ determines the collision time $\tau=l / v \sim R / E^{1 / 2}$ and the space diffusion rate $D \sim v l$. Let us assume that the friction is weak and many collisions take place during the dissipative time scale $1 / \gamma$. Then, at the equilibrium the diffusion in energy is compensated by dissipation so that the average energy is determined in a self-consistent way as

$$
E=v^{2} / 2 \sim\left(D_{E} / \gamma\right)^{1 / 2} \sim\left(l f^{2} / \gamma\right)^{2 / 3} \sim\left(f^{2} / \gamma\right)^{2 / 3}
$$

where in the last relation we used that in our case $l \sim R \sim 1$. The same expression can be also obtained in a more formal way by writing the relation for velocities $v_{n}, v_{n+1}$ between two consecutive collisions $n$ and $n+1$ from exact solution of the Newton equations. Then, assuming that the dynamics is ergodic and chaotic on the energy surface, the squares of these velocities may be averaged over all directions and times between collisions. In the equilibrium both average $v_{n}^{2}$ and $v_{n+1}^{2}$ are equal that gives the relation (1). The derivation of this expression assumes that many collisions take place. This requires that the amplitude of oscillations induced by radiation $a$ is larger then the distance between semi-disks: $a=f /\left(m \omega^{2}\right) \gg R$. Otherwise a trajectory drops on a simple attractor without scattering on semi-disks.

The numerical data are presented in Fig. 5 and confirm the dependence (11) in almost 4 orders of magnitude range. The deviations appear only at large values of $\gamma$ when the dissipation rate becomes comparable with the frequency of collisions. It is also interesting to note that 


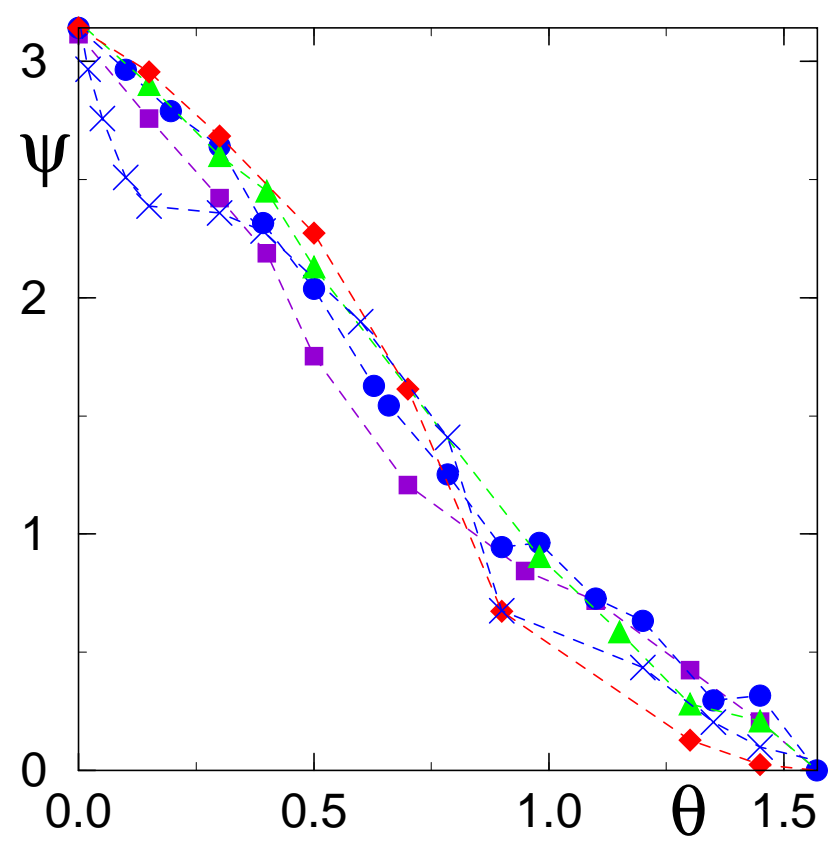

FIG. 4: (color online) Dependence of flow angle $\psi$ on polarization angle $\theta$, measured in radians, for $f=4$ and $\omega=1.5$ at $\gamma=0.2$ (squares), 0.1 (circles), 0.05 (triangles) and 0.025 (diamonds) and $\omega=0.1$ at $\gamma=0.1(\times)$. Dashed curves are drown to adapt an eye.

in agreement with (11) the dependence on radiation frequency $\omega$ is rather weak. Indeed, a change of $\omega$ by an order of magnitude gives only a factor of 2 change in $v^{2}$ and in the flow velocity $v_{f}$ (see Figs. 5,6). Even a larger change from $\omega=2.5$ to 0.025 at $f=4, \gamma=0.1$ gives only $30 \%$ increase of $v_{f}$ and $50 \%$ drop of $v^{2}$ (data not shown). Such a weak dependence on $\omega$ can be attributed to finite time correlations, existing in the chaotic dynamics, which have been neglected in the derivation of (11). The directionality of the flow is globally robust in respect to parameter variation (see Fig. 4). However, the dependence $\psi(\theta)$ has certain local changes for small $\theta$ when $\omega$ becomes smaller or comparable with $\gamma$.

The dependence of the velocity $v_{f}$ of the directed flow $\mathbf{v}_{f}$ is shown in Fig. 6 for $\theta=0$. The numerical data are well fitted by the relation:

$$
v_{f} \approx l^{2 / 3}\left(f \gamma / m^{2}\right)^{1 / 3} / 12\left(\gamma>\gamma_{c}\right) ; v_{f} \approx l \gamma / m\left(\gamma<\gamma_{c}\right)
$$

where $\gamma_{c} \approx(m f / l)^{1 / 2} / 40$ and we assumed $m=l=r_{d}=$ 1 . The dependence of $v_{f}$ on parameters for $\gamma>\gamma_{c}$ reminds the situation for a case of the Galton board with disks in presence of static force and friction (see [15, 18]). Indeed, assuming that the flow moves in a given direction its velocity can be estimated as $v_{f} \sim f \tau_{c} / m$ where $\tau_{c}=l / v$. According to (11) this leads to (2) for $\gamma>\gamma_{c}$. However, in a difference from [15, 18] the dependence is changed in the limit of small $\gamma \ll \gamma_{c}$. Qualitatively, one may say that in this limit the particle velocity becomes very large, the chaotic collisions become very often and

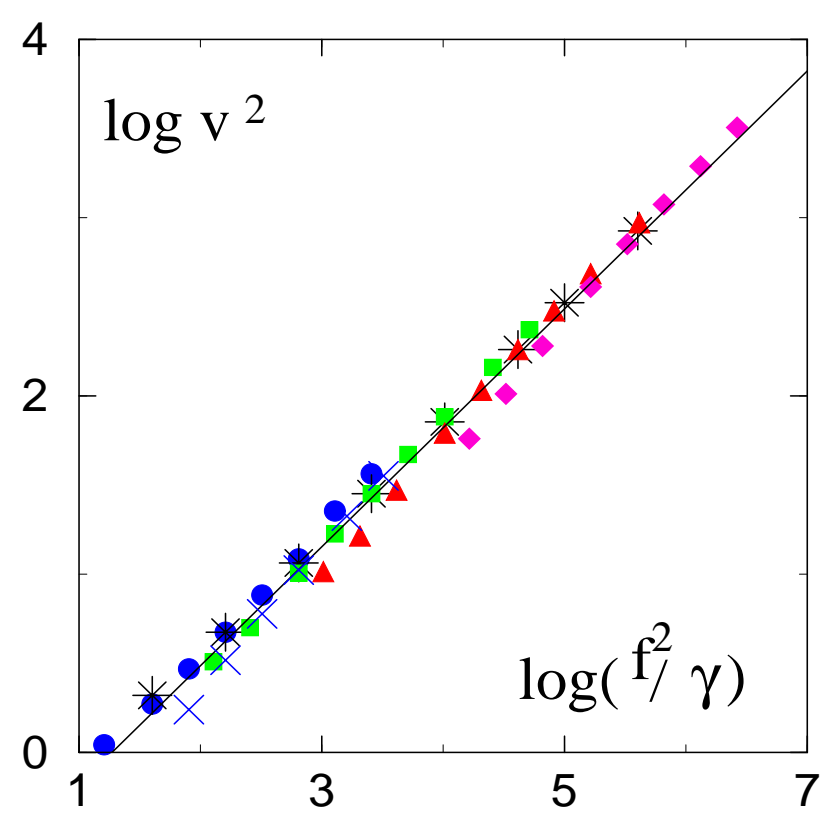

FIG. 5: (color online) Dependence of average $v^{2}$ on $f$ and $\gamma$ for $\theta=0$ at $\omega=1.5$ and $f=4$ (circles), 16 (squares), 64 (triangles), 256 (diamonds) and $\gamma=0.1(*)$; the case at $\omega=$ 0.1 and $f=4$ is shown by $(\times)$; total interval of parameters variation is $5 \cdot 10^{-3} \leq \gamma \leq 4,2 \leq f \leq 256$. The full line gives the dependence $v^{2}=\left(f^{2} / \gamma\right)^{2 / 3} / 7$. Logarithms are decimal here and in Fig. 6.

this leads to a significant averaging and decrease of $v_{f}$, which becomes proportional to the dissipation $\gamma$ that is responsible for appearance of directed flow. With the variation of $\theta$ from 0 to $\pi / 2$ the value of $v_{f}$ drops approximately by a factor 2 (e.g. for data in Fig. 4).

The results presented above show that the appearance of directed transport induced by polarized radiation is a robust phenomenon which is not very sensitive to the parameters of the model. Unfortunately we are not able to derive analytically the directionality dependence $\psi(\theta)$. Qualitatively, we may argue that for $\theta=0$ the dissipation leads to a trapping of particles in a funnel formed by semi-disks and the flow goes to the left. On the contrary, for $\theta=\pi / 2$ the vertical oscillations induced by radiation push particles out of a funnel and in presence of dissipation the flow goes to the right. This indicates that approximately $\psi=\pi-2 \theta$ in agreement with the numerical data. However, it is desirable to have a more rigorous derivation in addition to these handwaving arguments. We also note that in the case of charged particles an introduction of a magnetic field leads to a reorientation of the directed flow following approximately the direction given by the average Lorentz force (we leave a discussion of this effect for further studies).

The directed transport induced by polarized radiation demonstrates rather interesting and unusual properties and it would be interesting to study it in experi- 


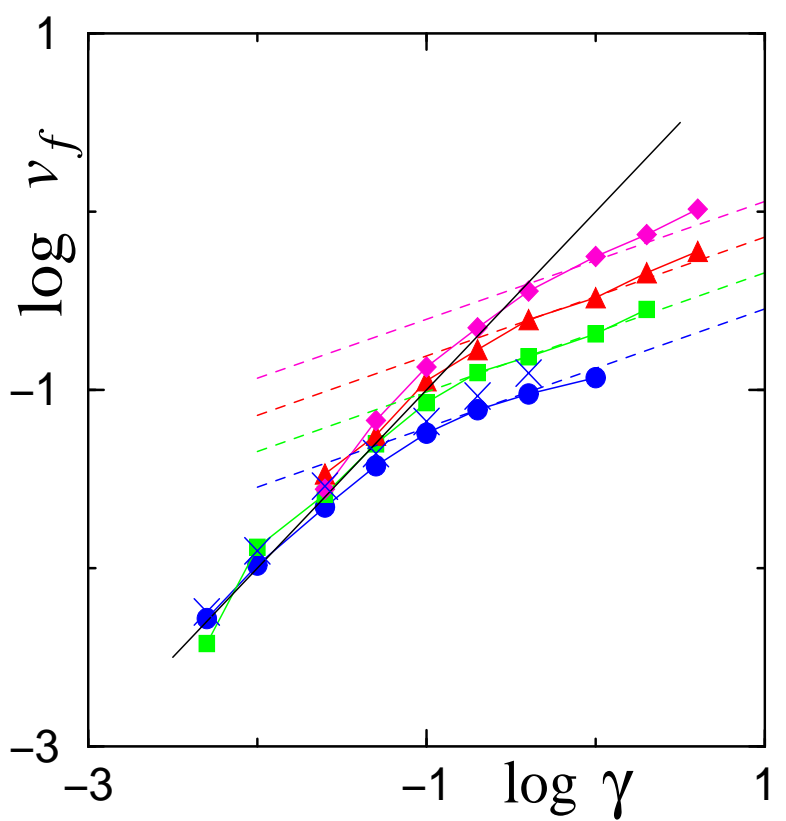

FIG. 6: (color online) Dependence of the flow velocity $v_{f}$ on friction $\gamma$ for $\theta=0$ and $\omega=1.5$ at $f=4,16,64$ and 256 and $\omega=0.1$ at $f=4$ (same symbols as in Fig. 5). The straight lines show the asymptotic behavior (2) at $\gamma>\gamma_{c}$ (dashed) and $\gamma<\gamma_{c}$ (full).

ments with semiconductor heterostructures. The nowadays technology allows to realize experimentally antidot superlattices (see e.g. [19]) and it is known that classical chaotic dynamics plays an important role in transport properties of such systems (see e.g. [20]). However, up to now only circular antidots have been studied. In fact technologically it is rather simple to replace disks by semi-disks and to place the whole board in a microwave field. Moreover, the effects of microwave radiation on transport in disordered mesoscopic systems have been studied experimentally [21] and it was shown that radiation may generate a directed transport in a case of broken space symmetry. Since disorder is symmetric in average it is clear that the ratchet effect should be significantly larger in artificially prepared asymmetric structures as the Galton board of semi-disks discussed here. A typical set of experimental parameters can be $r_{d} \sim R \sim l \sim 1 \mu \mathrm{m}, \omega / 2 \pi=1 \mathrm{GHz}, m=0.067 m_{e}$. For a microwave field of strength $\epsilon=10 \mathrm{~V} / \mathrm{cm}$ the amplitude of particle oscillations is $a=e \epsilon / m \omega^{2} \approx 60 \mu \mathrm{m}(a \gg R)$, so that the appearance of a simple attractor is excluded and a directed transport should appear due to presence of dissipative processes. A possibility to study transport in antidot lattices in presence of microwave radiation has been experimentally demonstrated in [22] and therefor the experimental realization of our model should be possible. Such experiments should allow to direct transport by radiation on mesoscopic scale and to study the dissipative processes in such systems from a new view point.
The theoretical model discussed here should be also further developed to be more adapted for such experiments (e.g. Fermi surface effects and more realistic dissipation should be taken into account).

We thank Kvon Ze Don for useful discussions.

* http://w3-phystheo.ups-tlse.fr/ dima

[1] R.D. Astumian and P. Hänggi, Physics Today 55 (11), 33 (2002).

[2] P. Reimann, Phys. Rep. 361, 57 (2002).

[3] F. Jülicher, A. Ajdari, and J. Prost, Rev. Mod. Phys. 69, 1269 (1997).

[4] H. Linke, T.E. Humphrey, A. Löfgren, A.O. Sushkov, R. Newbury, R.P. Taylor, and P. Omling, Science 286, 2314 (1999)

[5] J.B. Majer, J. Peguiron, M. Grifoni, M. Tusveld, and J.E. Mooij, Phys. Rev. Lett. 90, 056802 (2003).

[6] J.E. Villegas, S. Savel'ev, F. Nori, E.M. Gonzalez, J.V. Anguita, R. Garcia, and J.L. Vicent, Science 302, 1188 (2003).

[7] C. Mennerat-Robilliard, D. Lucas, S. Guibal, J. Tabosa, C. Jurczak, J.-Y. Courtois, and G. Grynberg, Phys. Rev. Lett. 82, 851 (1999).

[8] S. Matthias and F. Müller, Nature 424, 53 (2003).

[9] R. Eichhorn, P. Reimann, and P. Hänggi, Phys. Rev. Lett. 88, 190601 (2002).

[10] A rare study of finite dissipation case in one-dimention is given in J.L. Mateos, Phys. Rev. Lett. 84, 258 (2000); M.Barbi and M.Salerno, Phys. Rev. E 62, 1988 (2000).

[11] S. Flach, O. Yevtuschenko, and Y. Zolotaryuk, Phys. Rev. Lett. 84, 2358 (2000).

[12] H. Schanz, M.F. Otto, R. Ketzmerick, and T. Ditrich, Phys. Rev. Lett. 87, 070601 (2001).

[13] F. Galton, Natural inherritance, (Macmillan, London, 1889).

[14] I. P. Kornfeld, S. V. Fomin, and Ya. G. Sinai, Ergodic theory, (Springer, Berlin, 1982).

[15] A.D. Chepelianskii, and D.L. Shepelyansky, Phys. Rev. Lett. 87, 034101 (2001).

[16] In shops one can find a children toy board, placed between two plastic glasses, with disks replaced by same way oriented triangles and metallic balls moving on a board; shaking the board in $x$-axis moves all balls from right side of the board to the left. This corresponds to the case $\theta=0$ in Fig. 3.

[17] E. Ott, Chaos in dynamical systems, (Cambridge University Press, Cambridge, 1993).

[18] W. G. Hoover and B. Morgan, Chaos 2, 599 (1992).

[19] D. Weiss, M. L. Roukes, A. Menschig, P. Grambow, K. von Klitzing, and G. Weimann, Phys. Rev. Lett. 66, 2790 (1991).

[20] R. Fleischmann, T. Geisel, and R. Ketzmerick, Phys. Rev. Lett. 68, 1367 (1992); Europhys. Lett. 25, 219 (1994).

[21] A.A. Bykov, G.M. Gusev, Z.D.Kvon, D.I.Lubyshev, and V.P.Migal', Pis'ma Zh. Eksp. Teor. Fiz. 49, 13 (1989) [JETP Lett. 49, 13 (1989)].

[22] A.A. Bykov, G.M. Gusev, Z.D.Kvon, V.M.Kudryashev, and V.G.Plyukhin, Pis'ma Zh. Eksp. Teor. Fiz. 53, 407 (1991) [JETP Lett. 53, 427 (1991)]. 